\title{
Effect of beta-blockade on quantitative microvolt T-wave alternans in 24-hour continuous 12-lead ECG recordings in patients with long QT syndrome
}

\author{
Nobuhiro Takasugi $^{1}$ (D) | Mieko Takasugi ${ }^{2}$ | Hiroko Goto ${ }^{3}$ | Takashi Kuwahara ${ }^{3}$ | \\ Masanori Kawasaki $^{1}$ | Richard L. Verrier ${ }^{4}$ (D)
}

${ }^{1}$ Gifu University Hospital, Gifu, Japan

${ }^{2}$ Matsunami General Hospital, Gifu, Japan

${ }^{3}$ Gifu Prefectural General Medical Center, Gifu, Japan

${ }^{4}$ Department of Medicine, Harvard Medical School, Beth Israel Deaconess Medical

Center, Boston, Massachusetts

\section{Correspondence}

Nobuhiro Takasugi, Division of

Cardiovascular Medicine, Gifu University

Hospital, Gifu, Japan.

Email: sunanezumi417@yahoo.co.jp

Funding information

This research did not receive any specific grant from funding agencies in the public, commercial, or not-for-profit sectors.

\begin{abstract}
Background: The aim of study was to investigate effects of beta-blockade on microvolt T-wave alternans (TWA), a precursor of lethal arrhythmia, in patients with long QT syndrome (LQTS).

Methods: Eleven consecutive LQTS patients, types $1(n=6), 3(n=2)$, and "non-1, non-2, non-3" ( $n=3$ ) were enrolled. All patients underwent 24-hr continuous 12-lead ECG monitoring before and after initiation of beta-blockade therapy. TWA was measured using the modified moving average method.

Results: Seven (63.6\%) of the 11 patients studied were symptomatic, with history of cardiac arrest or documented Torsade de Pointes (TdP) in 4 and syncope in three patients. After a median follow-up of 34 months, beta-blockade reduced the number of symptomatic patients to 1 with $\operatorname{TdP}(p<0.02)$, in whom TdP frequency decreased from 25 events/60 months (0.42 event/month) to seven events/69 months ( 0.1 event/ month). In association with this reduction in symptoms, peak TWA decreased by $47 \%$ in the cohort after a median of eight months of beta-blockade therapy [from 95 (74-130) to 50 (39.5-64.5) $\mu \mathrm{V}, p=0.01$ ]. All patients exhibited TWA $\geq 42 \mu \mathrm{V}$ before beta-blockade therapy, which eliminated these episodes in four patients. Daily frequency of TWA $\geq 42 \mu \mathrm{V}$ episodes decreased by $87 \%$ [from $15(6-26)$ to $2(0-5)$ episodes/day, $p=0.009$ ]. Conclusions: This study is limited by the small sample size and is mainly hypothesis generating. TWA monitoring deserves further evaluation as a risk marker and a guide to therapy in LQTS patients in future large-scale studies.
\end{abstract}

\section{KEYWORDS}

beta-blockade, continuous 12-lead electrocardiography, modified moving average, long QT syndrome, microvolt T-wave alternans

\section{1 | INTRODUCTION}

Long QT syndrome (LQTS) is a genetic disorder characterized by prolonged ventricular repolarization and spatial dispersion of repolarization, both of which generate a vulnerable myocardial substrate promoting Torsade de Pointes (TdP) (JCS Joint Working
Group, 2012; Priori et al., 2013). Since the 1970 s, beta-blockade has been indicated as a first-line therapy in LQTS patients (JCS Joint Working Group, 2012; Moss, 1993; Priori et al., 2013; Schwartz, Crotti, \& Insolia, 2012; Schwartz, Periti, \& Malliani, 1975) to reduce syncope, TdP, and cardiac arrest but efficacy of beta-blockade to treat patients with LQTS differs by genotype (Abu-Zeitone, 

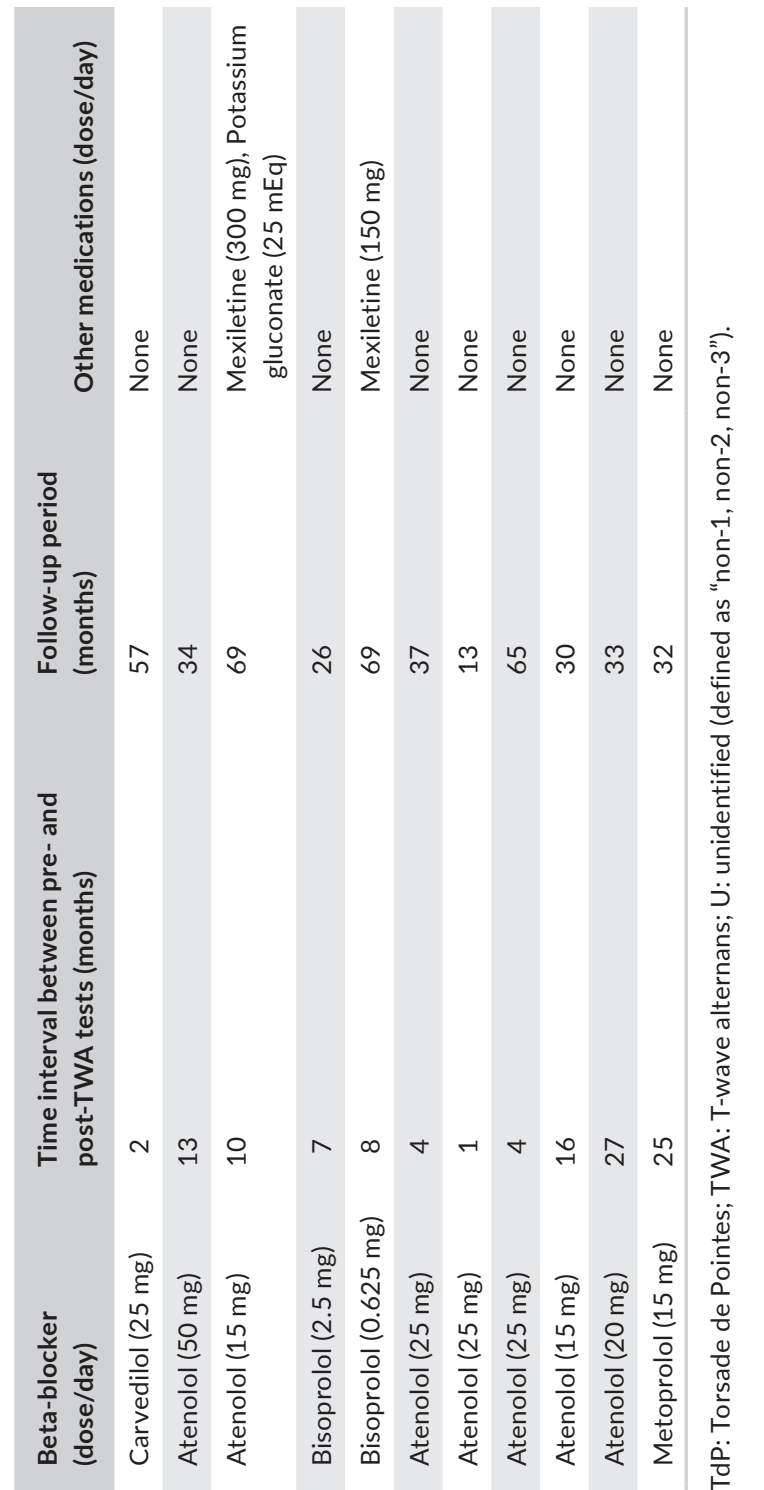


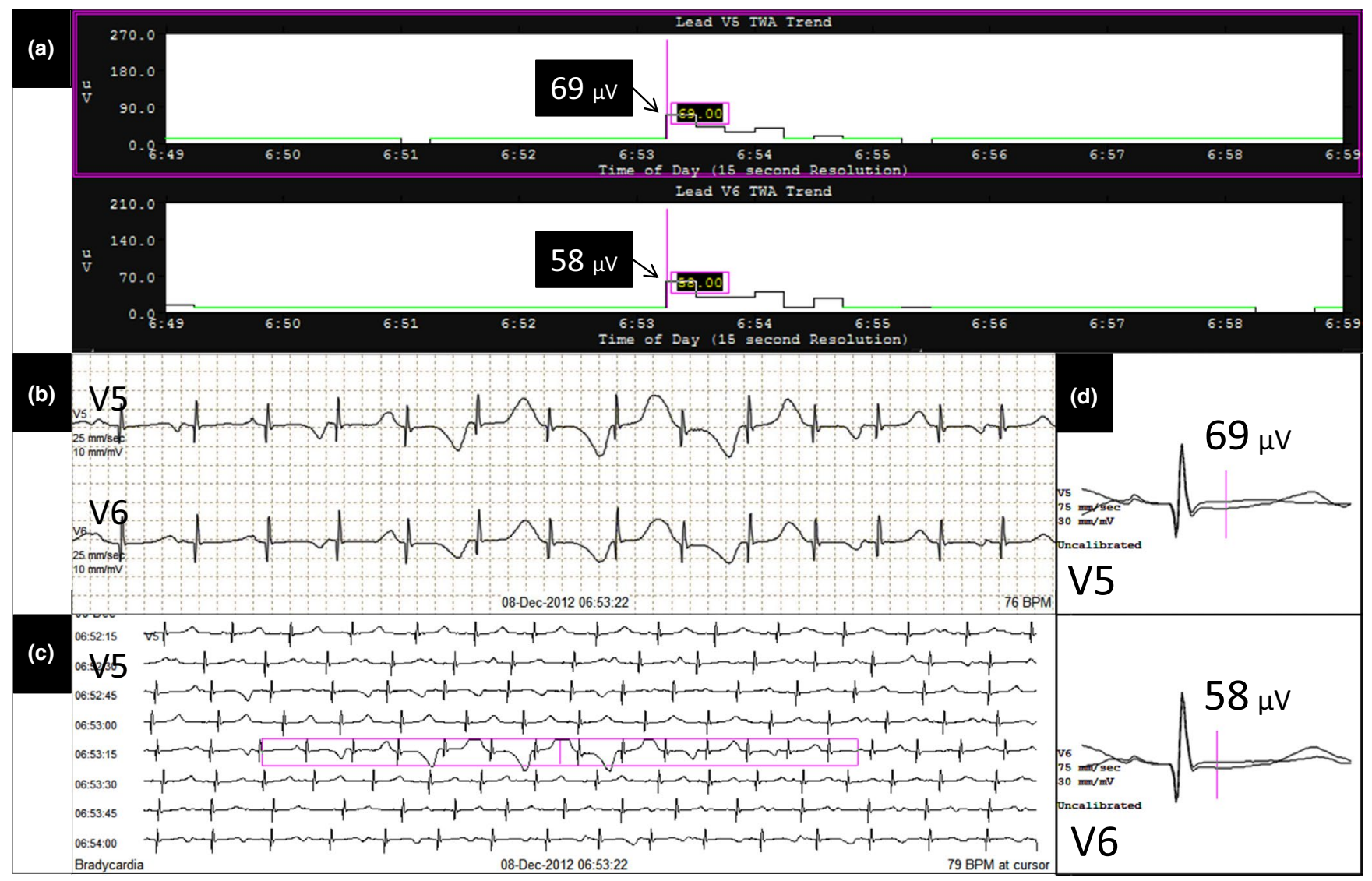

FIGURE 1 Heart-rate dependent T-wave alternans (TWA) episode from the representative patient 3 with long QT 3 and Torsade de Pointes. (a) TWA trend of leads V5 and V6 from 06:49 to 06:59 a.m. before beta-blockade therapy showing a transient TWA episode of $69 \mu \mathrm{V}$. ECG strips of leads V5 and V6 at 06:53, showing the TWA episode was visible and accompanied by alternating polarity of the T wave (b). Note that the TWA magnitude was progressively amplified or attenuated as the R-R interval became shorter or longer (c), suggesting heart-rate dependent mechanism. (D1-2) Templates of superimposed waveforms in leads V5 and V6 at 06:53

Peterson, Polonsky, McNitt, \& Moss, 2014; Ackerman et al., 2017; Chockalingam et al., 2012). Thus, indices for beta-blockade selection and dosage, identification of cases of noncompliance (Vincent et al., 2009), and referral for left cardiac sympathetic denervation (Schwartz et al., 2012) or implantable cardioverter-defibrillators to reduce risk for cardiac mortality are needed.

T-wave alternans (TWA), a beat-to-beat fluctuation in T-wave morphology and QT interval, is mechanistically linked to vulnerability to life-threatening ventricular tachyarrhythmias (Verrier et al., 2011; Verrier \& Ikeda, 2013) and may accentuate risk determined by QTc interval length, the initial diagnostic marker in LQTS patients (Moss, 1993). We recently reported that microvolt TWA $\geq 42 \mu \mathrm{V}$ measured in 24-hr continuous 12-lead ECGs provides $100 \%$ sensitivity for an association with TdP history (Takasugi et al., 2016). Although a few case reports demonstrated that beta-blockade reduces "macroscopic" TWA in association with arrhythmias in LQTS (Bosi, Cappato, Priori, \& Stramba-Badiale, 2002; Mache et al., 1996; Sugiyama et al., 2017), the effect of beta-blockade on microvolt levels of TWA in LQTS patients has not been systemically investigated.

The purpose of this study was (a) to investigate the effects of beta-blockade on peak TWA levels and frequency of microvolt TWA $\geq 42 \mu \mathrm{V}$ episodes in LQTS patients; (b) to classify the episodes of microvolt TWA $\geq 42 \mu \mathrm{V}$ into two categories based on the presence/ absence of heart-rate dependence; and (c) to assess the effects of beta-blockade according to heart-rate dependence.

\section{2 | METHODS}

\section{1 | Study patients}

A total of 11 consecutive LQTS patients were studied. A cardiac channel gene screen for LQTS-causing mutations in KCNQ1 (LQT1), KCNH2 (LQT2), and SCN5A (LQT3) using direct sequencing (Sanger method) was performed in all patients at National Cerebral and Cardiovascular Center (Osaka, Japan). Unidentified LQT type was defined as "non-1, non-2, non-3" LQT type. All unidentified LQTS patients enrolled in this study fulfilled the diagnostic criteria for LQTS (score $\geq 3.5$ ) (Priori et al., 2013). The participants were enrolled between December 2012 and August 2017 at Gifu University Hospital and Gifu Prefectural General Medical Center (Gifu, Japan). The study protocol conformed to the ethical guidelines of the 1975 Declaration of Helsinki as reflected in a priori approval by the institution's human research committee. Informed consent was obtained from each patient. 


\section{2 | Twenty-four-hour 12-lead continuous ECG monitoring}

All patients underwent 24-hr continuous 12-lead ECG monitoring (SEER 12 Ambulatory Recorder, GE Healthcare, Milwaukee, WI, USA) before and after initiation of beta-blockade therapy. Careful skin preparation and high-resolution electrodes (Blue Sensor L, Ambu A/S, Ballerup, Denmark) were used to minimize noise. Average heart rate across the $24-\mathrm{hr}$ recording was automatically calculated. The maximum QT interval within the first minute of supine rest was taken as the index value. The QT interval was automatically measured in lead II or $\mathrm{V}_{5}$ (whichever was longer) every $15 \mathrm{~s}$ using MARS Holter Analysis Workstation Software Version 8 (GE Healthcare), which was verified manually. Bazett's formula was then applied.

\section{3 | Measurement of microvolt TWA}

Microvolt TWA levels were calculated by the time-domain modified moving average method (Nearing \& Verrier, 2002) using MARS Holter Analysis Workstation Software Version 8 (GE Healthcare). The data were visually inspected and scored by one clinical technologist who was blinded to the patient information. TWA levels from recordings with heart rate $>120 \mathrm{bpm}$ or noise levels $>20 \mu \mathrm{V}$ were excluded from the analysis. Peak TWA in the 12 leads and frequency of TWA $\geq 42 \mu \mathrm{V}$ episodes were determined before and after initiating beta-blockade therapy. We monitored the frequency of TWA episodes $\geq 42 \mu \mathrm{V}$, which provided $100 \%$ sensitivity for an association with TdP history in LQTS patients in our prior study (Takasugi et al., 2016).

\section{4 | Classification of microvolt TWA $\geq 42 \mu \mathrm{V}$ episodes}

The TWA $\geq 42 \mu \mathrm{V}$ episodes were categorized based on heart-rate dependence. TWA episodes that were triggered when the TQ interval was $<200 \mathrm{~ms}$ [i.e., during short diastolic interval (DI)] were considered heart-rate dependent. A premature impulse with a DI <200 ms may shorten action potential duration in the human ventricle (Taggart et al., 2003). Thus, a short DI may facilitate heart-rate-dependent TWA in the presence of transmural dispersion of repolarization (Shimizu \& Antzelevitch, 1999; Takasugi et al., 2018). Heart-rate-independent TWA was defined as TWA episodes that were triggered when the TQ interval was $>200$ ms (i.e., during long DI).

\section{5 | Beta-blocker therapy}

Beta-blocker dose was increased according to body weight gain (or the maximum tolerated dose) after the first assessment of TWA in pediatric patients.

\section{6 | Statistical analysis}

Statistical analyses were performed using EZR (Saitama Medical Center, Jichi Medical University, Saitama, Japan), which is a graphical user interface for $\mathrm{R}$ (The R Foundation for Statistical Computing, Vienna, Austria). Continuous variables were presented as median (interquartile range). Peak TWA levels and frequency of TWA $\geq 42 \mu \mathrm{V}$ episodes before and after initiating beta-blockade were compared using the Wilcoxon signed-rank test. Fisher's exact test was used for reduction in patients with symptoms. All

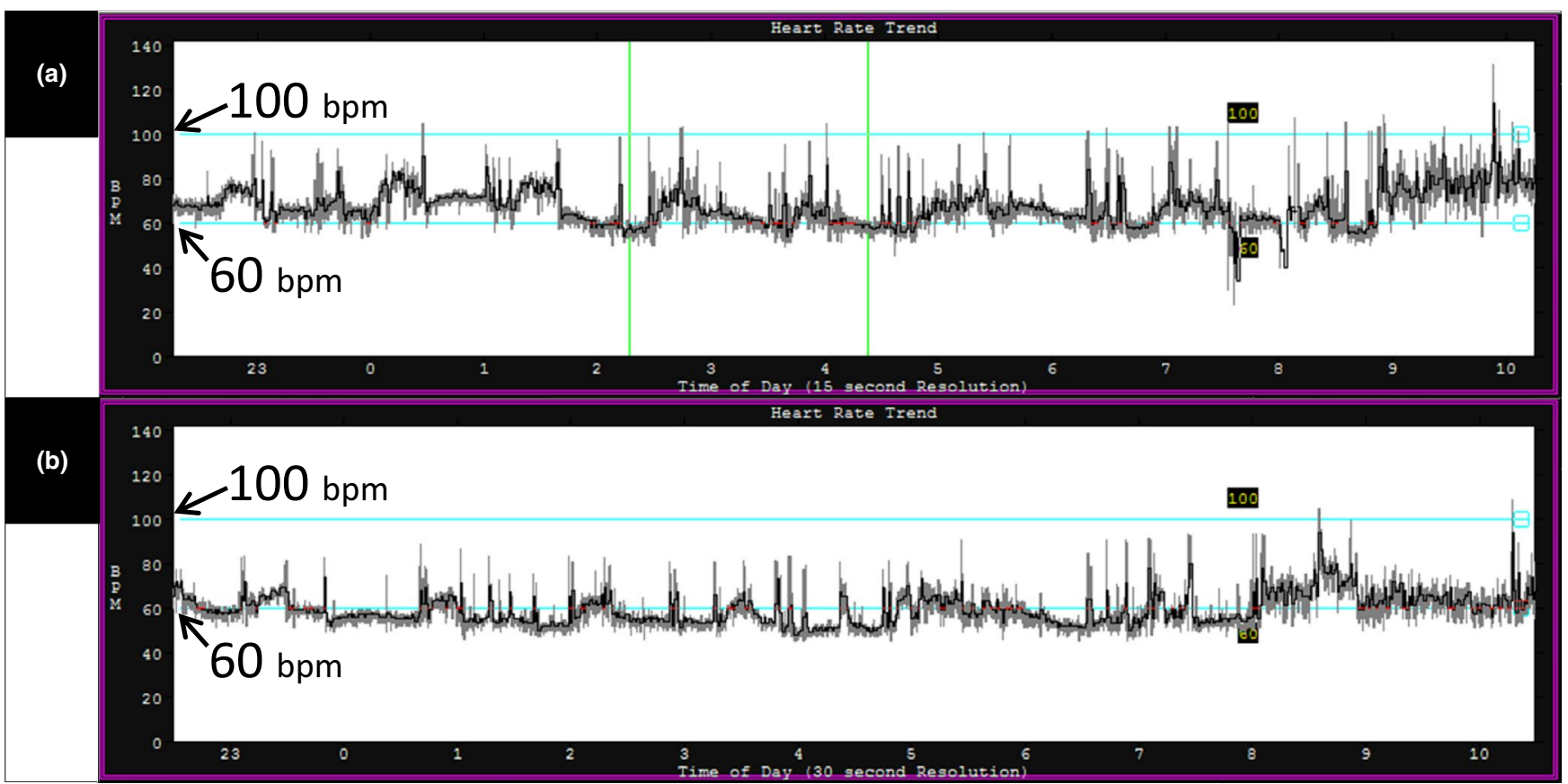

FIGURE 2 Heart rate trend from 22:00 to 10:00 before (a) and after (b) beta-blockade therapy of patient 3 with long QT3 and Torsade de Pointes (the same patient as in figure 1). Note the decrease in both baseline heart rate and frequency of transient tachycardia (>100 bpm) episodes 
significant tests were two-sided. Results were considered statistically significant when the $P$-value was $<0.05$.

\section{3 | RESULTS}

\section{1 | Patient characteristics}

The clinical characteristics of the 11 patients (seven males and four females) with LQTS types 1 (LQT1, $n=6$ ), 3 (LQT3, $n=2$ ), and "non-1, non-2, non-3" $(n=3)$ are summarized in Table 1 . Median age at enrollment was 12 (7-17.5) years. The beta-blocker dosage indicated was at the time of the second TWA test. Of the 11 patients studied, 7 (63.6\%) were symptomatic: four patients had experienced cardiac arrest or documented TdP and the other 3 had experienced syncope prior to enrollment and initiation of beta-blockade. In the two patients with LQT3 (patients 3 and 5), beta-blockade was added to mexiletine. Specifically, patient 3 with LQT3 experienced recurrent episodes of TdP recorded via implantable cardioverter-defibrillator (ICD) telemetry.

\section{2 | Results of 24-hr continuous 12-lead ECG analysis in LQTS patients}

Results of continuous 12-lead ECG analysis before and after initiating beta-blockade therapy are summarized in Table 2. There was a difference between time interval to TWA reassessment and clinical follow-up period. The time interval between the first and the second

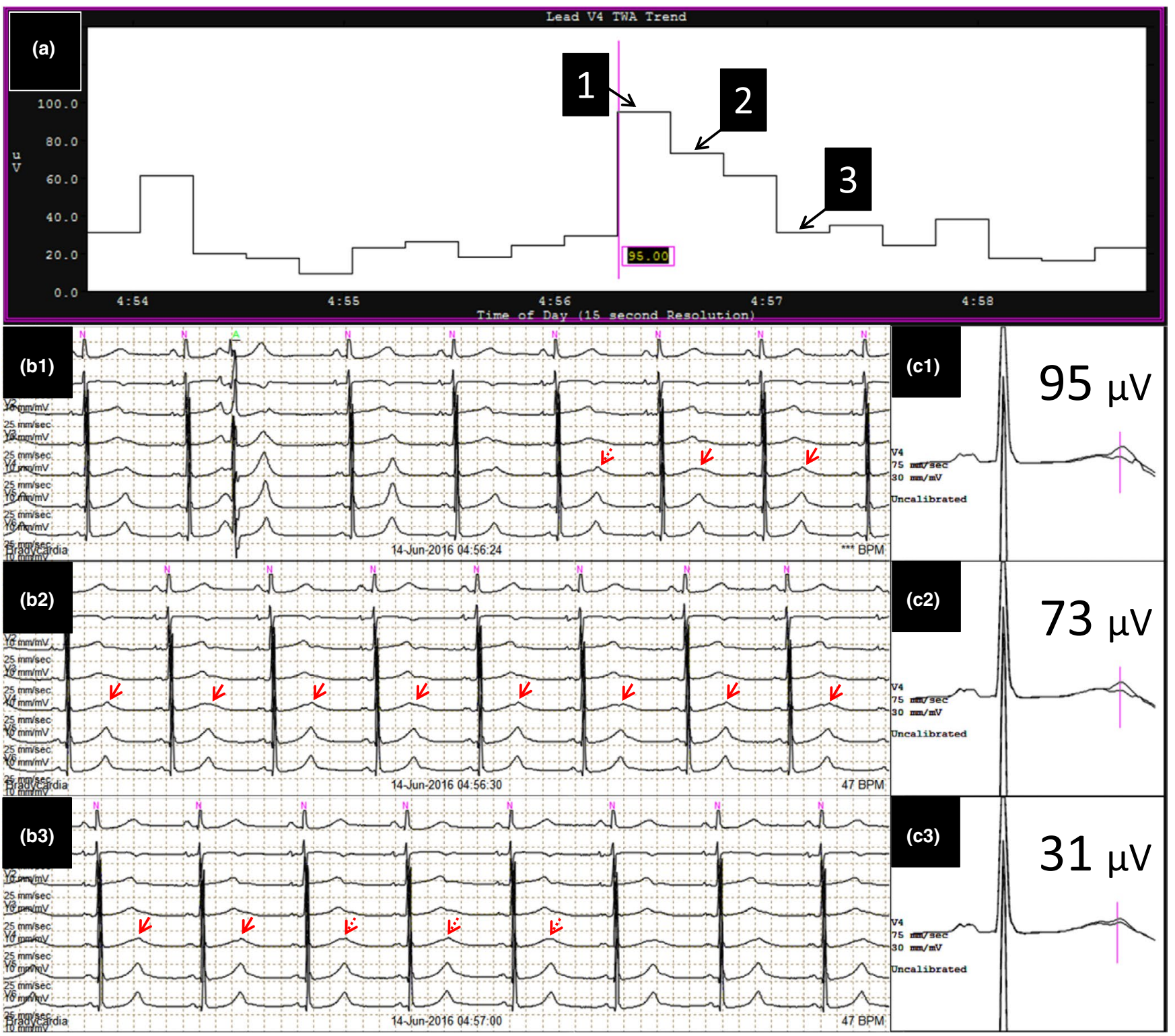

FIG URE 3 Heart-rate independent T-wave alternans (TWA) episode recorded in patient 4 with "non-1, non-2, non-3" long QT and Torsade de Pointes. (a) TWA trend in lead V4 (04:54-04:58 a.m.) before beta-blockade therapy showing a transient episode of TWA ( $95 \mu \mathrm{V}$ ) at 04:56. (B1-3) ECG strips at points 1-3 in the TWA trend (from top to bottom; leads II, V1-V6) showing that the TWA was slightly visible in lead V4 (red arrows). Note that TWA appeared during bradycardia (47 bpm) and the episode was provoked or terminated without any change in the heart rate. (C1-3) Templates of superimposed waveforms in lead V4 for TWA values at points 1-3 in the TWA trend 


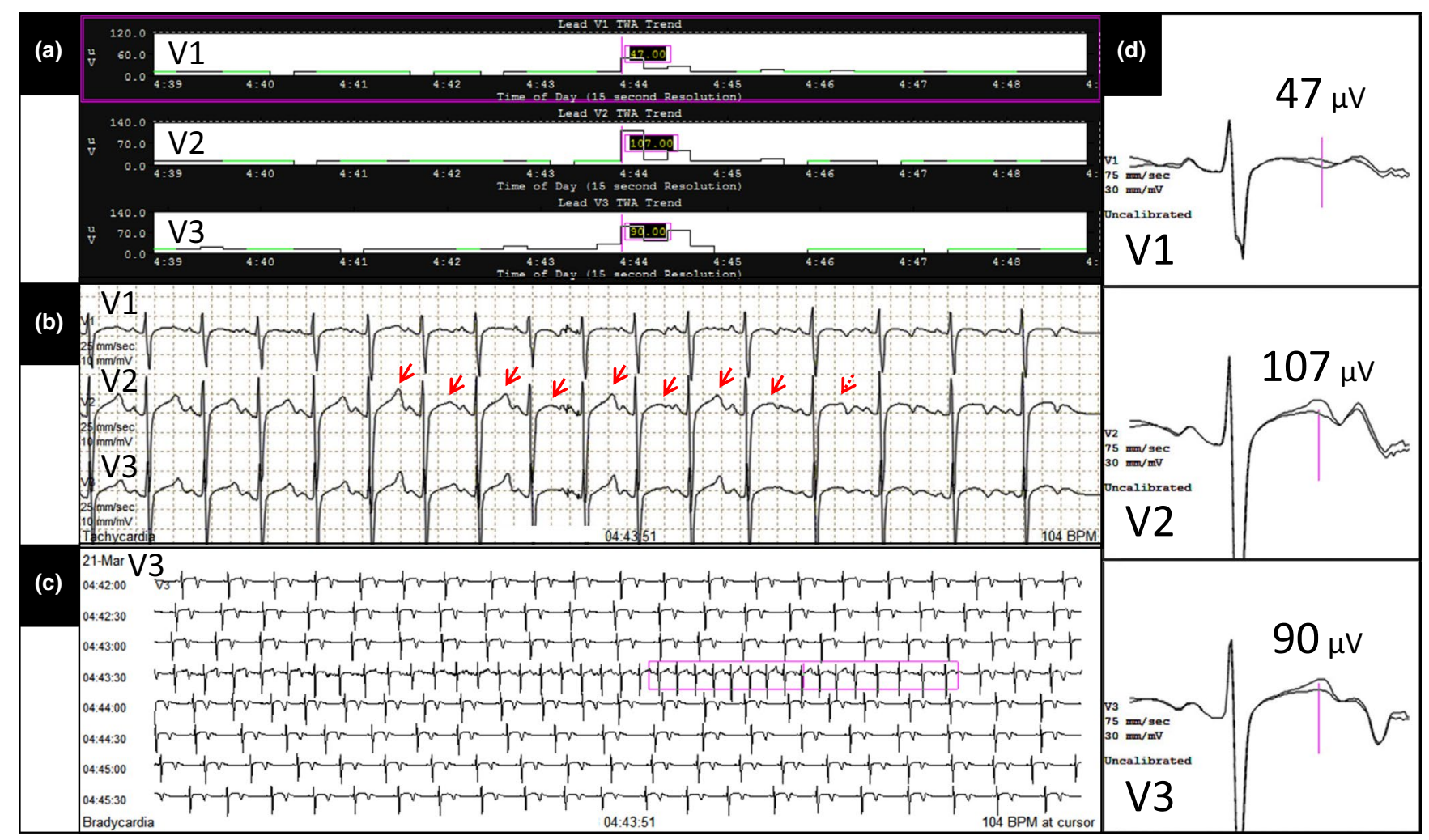

FIGURE 4 Heart-rate dependent T-wave alternans (TWA) episode from the representative patient 5 with long QT3 and syncope. (a) TWA trend of leads V1-3 from 04:39 to 04:49 a.m. before beta-blockade therapy showing a transient TWA episode of $107 \mu \mathrm{V}$. ECG strips of leads V1-3 at 06:53, showing the TWA episode was visible (red arrows) (b). Note that the TWA magnitude was progressively amplified or attenuated as the R-R interval became shorter or longer (c), suggesting heart-rate dependent mechanism. (d) Templates of superimposed waveforms in leads V1-3 at 04:43

TWA tests was a median of 8 (4-16) months. After a median followup of 34 months, beta-blockade reduced the number of symptomatic patients to 1 with TdP (patient 3) $(p<0.02$ ), in whom TdP frequency decreased from 25 events (including 5 ICD shocks)/60 months before beta-blocker initiation to seven events (including 3 ICD shocks)/69 months after beta-blocker initiation (Table 2). There was no significant change in QTc after the therapy [before vs. after, 479 (455-500.5) vs. 484 (471.5-506.5) $\mu \mathrm{V}, p=0.36]$. Average heart rate for $24 \mathrm{hr}$ significantly decreased from $77(70.5-83.5)$ to 67 (66-76) bpm ( $p=0.01)$ after the therapy.

Figure 1 shows results of continuous 12-lead ECG from patient 3 with LQT3 and TdP, in whom multiple transient episodes of TWA were provoked or terminated by sudden R-R interval shortening or prolongation in the morning. The TWA magnitude was progressively amplified or attenuated (1-B) as the R-R interval became shorter or longer (1-C), suggesting a heart-rate-dependent mechanism. The addition of atenolol to mexiletine reduced both baseline heart rate and frequency of transient tachycardia episodes (Figure 2). In this patient, in association with the reduction in TdP events, both peak TWA level and frequency of TWA $\geq 42 \mu \mathrm{V}$ episodes were decreased after the therapy, although TWA remained severely abnormal at $162 \mu \mathrm{V}$ (Table 2). Figure 3 shows a heartrate-independent TWA episode observed in patient 4 with "non-1, non-2, non-3" LQTS and TdP. TWA appeared during bradycardia
(47 bpm) without any change in heart rate and alternation occurred in the terminal portion of the $T$ waves (2-B). In this patient, betablockade abolished TWA $\geq 42 \mu \mathrm{V}$ episodes as it reduced peak TWA to $19 \mu \mathrm{V}$ (Table 2). Both heart-rate-dependent and heart-rate-independent TWA were observed at different times of the day in 3 (27.3\%) patients (Table 2). Representative patient 5 exhibited both heart-rate-dependent (Figure 4) and heart-rate-independent TWA (Figure 5).

\subsection{Peak TWA levels and frequency of $\geq 42 \mu \mathrm{V}$ TWA episodes before and after therapy}

In association with the reduction in symptoms, peak TWA level decreased by $47 \%$ [before vs. after, 95 (74-130) vs. 50 (39.5-64.5) $\mu \mathrm{V}, p=0.01]$, although remaining abnormal in seven patients (Table 2). All patients exhibited TWA $\geq 42 \mu \mathrm{V}$ episodes before beta-blockade therapy, which completely eliminated these episodes in 4 (36.4\%) patients. In addition, daily frequency of TWA $\geq 42 \mu \mathrm{V}$ episodes was reduced by $87 \%$ [before vs. after, 15 (6-26) vs. $2(0-5), p=0.009]$. Beta-blockade significantly reduced the incidence of heart-rate-dependent TWA $\geq 42 \mu \mathrm{V}$ episodes from 6 $(3.5-20)$ to $0(0-2.5)$ episodes/day $(p=0.03)$ and halved the number of patients with heart-rate-dependent TWA $\geq 42 \mu \mathrm{V}$ episodes from 10 to 5 of the 11 patients studied. The reduction in incidence 


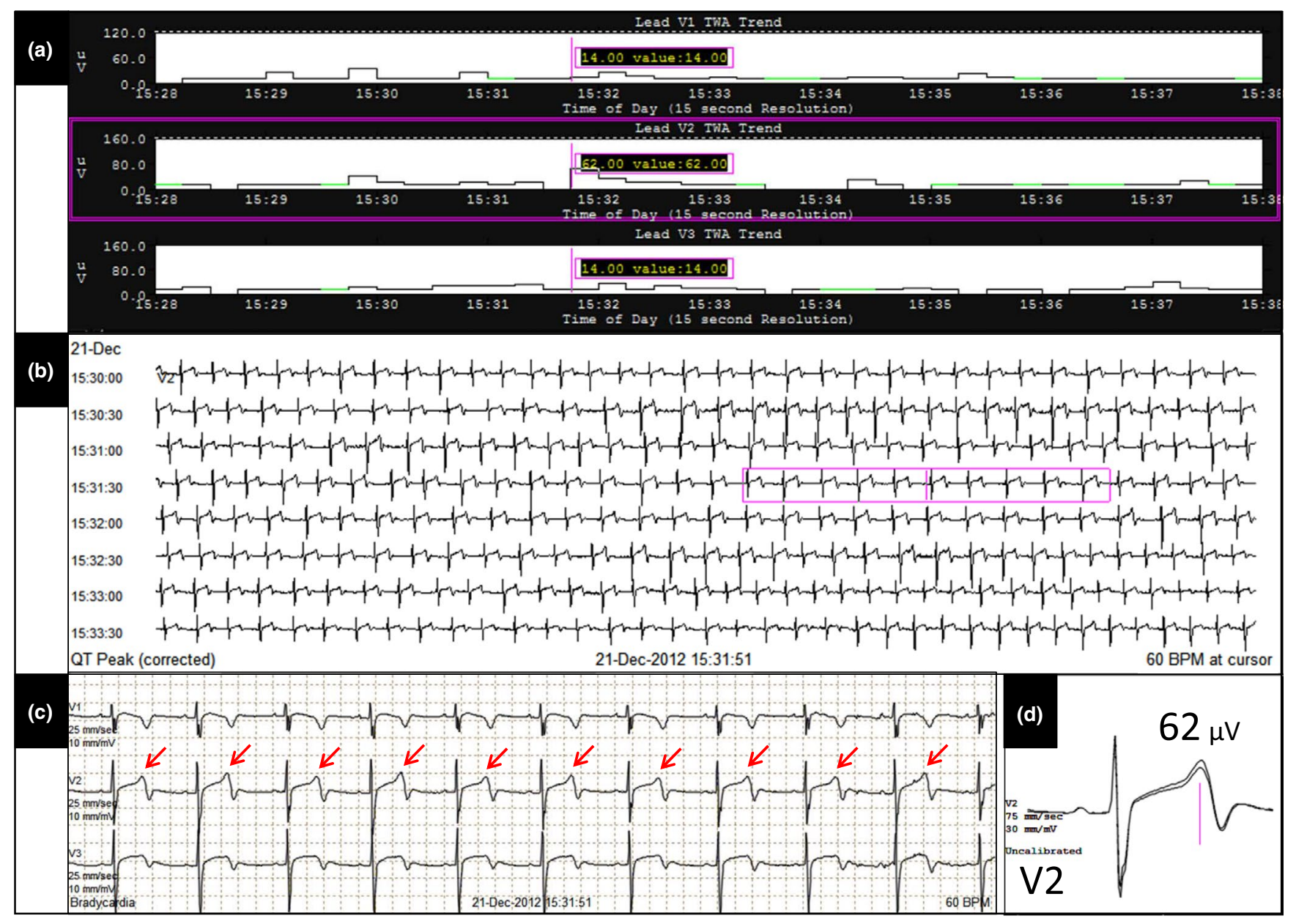

FIGURE 5 Heart-rate independent T-wave alternans (TWA) episode observed in the patient 5 with long QT3 and syncope (the same patient as in figure 4). (a) TWA trend of leads V1-3 from 15:28 to 15:38 before beta-blockade therapy showing a transient TWA episode of $62 \mu \mathrm{V}$. (b) ECG strips of lead V2 from 15:30 to 15:33, demonstrating that the TWA episode was provoked or terminated without any change in the heart rate. (c) ECG strips of leads V1-3 showing that the TWA episode was slightly visible in lead V2. (d) Templates of superimposed waveforms in lead V2 at 15:31

of heart-rate-independent TWA $\geq 42 \mu \mathrm{V}$ episodes/day was not significant [before vs. after, $0(0-6)$ vs. $0(0-0), p=0.1]$, but the number of patients with heart-rate-independent TWA $\geq 42 \mu \mathrm{V}$ episodes also decreased from 5 to 2 among the 11 patients studied (Table 2).

\subsection{Heart rate at maximum heart-rate- dependent and heart-rate-independent TWA episodes before and after therapy}

There was no significant difference in heart rate at the maximum heart-rate-dependent TWA before and after therapy among the 10 patients who exhibited heart-rate-dependent TWA [109 (106-111.5) bpm before therapy vs. 107.5 (103.5-112.75) bpm after therapy, $p=0.6]$. Also, there was no significant difference in heart rate at the maximum heart-rate-independent TWA before and after therapy among the five patients who exhibited heart-rate-independent TWA [56 (50-58) bpm before therapy vs. 57 (53-58) bpm after therapy, $p=0.8]$.

\section{4 | DISCUSSION}

\section{1 | Effect of beta-blockade on quantitative microvolt TWA in LQTS patients}

In the present study, we have shown that beta-blockade therapy significantly reduces both peak TWA level and frequency of TWA $\geq 42 \mu \mathrm{V}$ episodes in association with the reduction in syncope and $\mathrm{TdP}$, raising the possibility that TWA may serve as a marker of risk for cardiac events in LQTS patients. This study included two male patients with type 3 LQTS (patients 3 and 5), in whom effectiveness of beta-blockade remains unclear (Wilde et al., 2016). Peak TWA was reduced from 285 to $162 \mu \mathrm{V}$ and TWA episodes $\geq 42 \mu \mathrm{V}$ from 211 to 115 in patient 3 in association with a decrease in TdP frequency. More aggressive treatment of the residual TWA such as by left cardiac sympathetic denervation may be required in this patient. Beta-blockade therapy abolished syncope in patient 5 while reducing peak TWA from 119 to $44 \mu \mathrm{V}$, near the cutpoint of abnormality for LQTS patients (Takasugi et al., 2016). 


\subsection{TWA and arrhythmia risk in LQTS patients}

The effect of beta-blockade on quantitative microvolt TWA has not previously been investigated in LQTS patients, although a few cases of reduction or elimination of macroscopic TWA by betablockade and improvement in symptoms in pediatric LQTS patients have been reported (Bosi et al., 2002; Mache et al., 1996; Sugiyama et al., 2017). Japanese Circulation Society guidelines recommend microvolt TWA for prediction of sudden cardiac death in patients with ischemic (JCS Joint Working Group, 2012; Klingenheben, Grönefeld, Li, \& Hohnloser, 2001; Rashba et al., 2002) or non-ischemic cardiomyopathy (JCS Joint Working Group, 2012; Klingenheben et al., 2001); in these populations, metoprolol (Klingenheben et al., 2001), d,l-sotalol (Klingenheben et al., 2001), and esmolol (Rashba et al., 2002) have been reported to suppress microvolt TWA along with arrhythmia (Verrier \& Ikeda, 2013). Beta-blockade is standard-of-care therapy for LQTS patients. However, the efficacy of beta-blockade in LQTS patients may vary by genotype (Abu-Zeitone et al., 2014; Ackerman et al., 2017; Chockalingam et al., 2012). Thus, there is need to investigate the potential of TWA to serve as a therapeutic target.

Stimulation of the left stellate ganglion elicits TWA in experimental models of LQTS (Schwartz \& Malliani, 1975), and stellectomy abolishes TWA in LQTS patients (Crampton, 1979; Priori et al., 2013). Vagus nerve stimulation reduces microvolt TWA amplitude in patients with epilepsy (Schomer, Nearing, Schachter, \& Verrier, 2014) and heart failure (Libbus, Nearing, Amurthur, KenKnight, \& Verrier, 2016), presumably through its well-established anti-adrenergic effects. This clinical and experimental evidence of reduction in TWA by beta-adrenergic blockade can provide an explanation for our findings.

\section{3 | Types and mechanisms of microvolt TWA}

This study demonstrated that there may be two different types of TWA in LQTS patients, heart-rate-dependent and heart-rate-independent TWA. Several mechanisms of TWA have been demonstrated and linked to arrhythmogenesis in different diseases (Qu, Xie, Garfinkel, \& Weiss, 2010). In LQTS patients, there are two distinct mechanisms of TWA: (a) TWA elicited by short DI in the presence of transmural dispersion of action potentials or restitution kinetics (i.e., heart-rate-dependent TWA) (Shimizu \& Antzelevitch, 1999; Takasugi et al., 2018) and (b) TWA induced by 2:1 early afterdepolarizations, which usually emerge during bradycardia (i.e., heart-rate-independent TWA) (Qu et al., 2010). Both of these arrhythmogenic mechanisms may play a role in initiating TdP (Qu et al., 2010).

\section{4 | Effect of beta-blockade on TWA according to heart-rate dependency}

Although beta-blockade can suppress the both heart-rate-dependent and heart-rate-independent TWA (Qu et al., 2010), our results indicate that the efficacy of beta-blockade may vary with heartrate dependence. Specifically, beta-blockade significantly reduced heart-rate-dependent TWA episodes $(p=0.03)$, but heart-rate-independent TWA episodes did not decrease significantly $(p=0.2)$. There was no significant difference in heart rate at maximum level of heartrate-dependent TWA before and after beta-blockade. These facts indicate that LQTS patients with dispersion of repolarization can exhibit heart-rate-dependent TWA when the diastolic interval suddenly shortens and approaches zero, even after beta-blockade. Beta-blocker can suppress both magnitude and frequency of TWA by reducing not only baseline heart rate but also sudden increase in heart rate (as in Figure 2). Although beta-blocker can suppress early afterdepolarizations, a possible cause of heart-rate-independent TWA, our results indicate that heart-rate-independent TWA may include other mechanisms.

\subsection{Clinical implications}

Higher microvolt TWA magnitudes indicate increased risk for ventricular tachyarrhythmias (Verrier et al., 2011). Thus, quantitative microvolt TWA monitoring might help not only to assess arrhythmia risk but also to guide therapy and to identify cases of noncompliance (Vincent et al., 2009) in LQTS patients. It would be valuable to investigate the efficacy of beta-blockade in reducing microvolt TWA according to LQT genotype and/or beta 1 -receptor selectivity (Abu-Zeitone et al., 2014; Chockalingam et al., 2012).

\section{6 | Limitations}

First, the sample size of this study was small because of the low prevalence of the disease and thus should be considered hypothesis generating rather than confirmatory. Second, only a single 24-hr monitoring session was performed before and after initiation of beta-blockade, and reproducibility of the findings was not assessed. The availability of ECG patch monitors, which have been shown to be useful in patients with LQT syndrome could provide the opportunity for more comprehensive monitoring (Castelletti et al., 2018; Verrier, 2018).

\section{5 | CONCLUSIONS}

Beta-blockade therapy reduced both the frequency of TWA $\geq 42 \mu \mathrm{V}$ episodes and the peak amplitude of TWA in 11 LQTS patients in association with reductions in incidence of syncope and TdP. Moreover, this study indicates that there are two different types of TWA in LQTS patients, heart-rate-dependent and heart-rate-independent TWA and that the efficacy of beta-blockade may vary with heart-rate dependence. These observations deserve study in a larger population of LQTS patients, to determine whether TWA could track efficacy of beta-blockade to reduce cardiac events and to identify beta-blockade noncompliance.

\section{ACKNOWLEDGMENTS}

R.L.V. receives royalty income from Georgetown University and Beth Israel Deaconess Medical Center for intellectual property on 
the modified moving average method of TWA analysis, which has been licensed by GE Healthcare and was used in this study.

\section{CONFLICT OF INTEREST}

The other authors have declared no conflicts of interest.

\section{AUTHORS' CONTRIBUTIONS}

N.T. was involved in concept/design, data collection, data analysis/ interpretation, and drafting the article; M.T. and R.L.V. were involved in concept/design, data analysis/interpretation, and critical revision of the article; H.G. and T.K. were involved in data collection and critical revision of the article; M.K. was involved in data analysis, statistics, and critical revision of the article and funding.

\section{ORCID}

Nobuhiro Takasugi (iD https://orcid.org/0000-0001-6194-7490

Richard L. Verrier (iD https://orcid.org/0000-0001-5602-6793

\section{REFERENCES}

Abu-Zeitone, A., Peterson, D. R., Polonsky, B., McNitt, S., \& Moss, A. J. (2014). Efficacy of different beta-blockers in the treatment of long QT syndrome. Journal of the American College of Cardiology, 64, 13521358. https://doi.org/10.1016/j.jacc.2014.05.068

Ackerman, M. J., Priori, S. G., Dubin, A. M., Kowey, P., Linker, N. J., Slotwiner, D., ... Gold, M. R. (2017). Beta-blocker therapy for long QT syndrome and catecholaminergic polymorphic ventricular tachycardia: Are all beta-blockers equivalent? Heart Rhythm: the Official Journal of the Heart Rhythm Society, 14, e41-e44.

Bosi, G., Cappato, R., Priori, S. G., \& Stramba-Badiale, M. (2002). Complex electrocardiographic findings in a neonate with long QT syndrome. Italian Heart Journal, 3, 605-607.

Castelletti, S., Dagradi, F., Goulene, K., Danza, A. I., Baldi, E., StrambaBadiale, M., \& Schwartz, P. J. (2018). A wearable remote monitoring system for the identification of subjects with a prolonged QT interval or at risk for drug-induced long QT syndrome. International Journal of Cardiology, 266, 89-94. https://doi.org/10.1016/j. ijcard.2018.03.097

Chockalingam, P., Crotti, L., Girardengo, G., Johnson, J. N., Harris, K. M., van der Heijden, J. F., ... Wilde, A. A. (2012). Not all beta-blockers are equal in the management of long QT syndrome types 1 and 2: Higher recurrence of events under metoprolol. Journal of the American College of Cardiology, 60, 2092-2099. https://doi.org/10.1016/j. jacc.2012.07.046

Crampton, R. (1979). Preeminence of the left stellate ganglion in the long QT syndrome. Circulation, 59, 769-778. https://doi.org/10.1161/01. CIR.59.4.769

JCS Joint Working Group (2012). Guidelines for risks and prevention of sudden cardiac death (JCS 2010): - digest version -. Circulation Journal, 76, 489-507. https://doi.org/10.1253/circj. CJ-88-0022

Klingenheben, T., Grönefeld, G., Li, Y. G., \& Hohnloser, S. H. (2001). Effect of metoprolol and d, l-sotalol on microvolt-level T-wave alternans. Results of a prospective, doubleblind, randomized study. Journal of the American College of Cardiology, 38, 2013-2019. https:// doi.org/10.1016/S0735-1097(01)01661-8
Libbus, I., Nearing, B. D., Amurthur, B., KenKnight, B. H., \& Verrier, R. L. (2016). Autonomic regulation therapy suppresses quantitative Twave alternans and improves baroreflex sensitivity in patients with heart failure enrolled in the ANTHEM-HF study. Heart Rhythm: the Official Journal of the Heart Rhythm Society, 13, 721-728. https://doi. org/10.1016/j.hrthm.2015.11.030

Mache, C. J., Beitzke, A., Haidvogl, M. Jr, Gamillscheg, A., Suppan, C., \& Stein, J. I. (1996). Perinatal manifestations of idiopathic long QT syndrome. Pediatric Cardiology, 17, 118-121. https://doi.org/10.1007/ BF02505096

Moss, A. J. (1993). Measurement of the QT interval and the risk associated with QTc interval prolongation: A review. American Journal of Cardiology, 72, 23B-25B. https://doi. org/10.1016/0002-9149(93)90036-C

Nearing, B. D., \& Verrier, R. L. (2002). Modified moving average analysis of T-wave alternans to predict ventricular fibrillation with high accuracy. Journal of Applied Physiology, 92, 541-549. https://doi. org/10.1152/japplphysiol.00592.2001

Priori, S. G., Wilde, A. A., Horie, M., Cho, Y., Behr, E. R., Berul, C., ... Tracy, C. (2013). HRS/EHRA/APHRS expert consensus statement on the diagnosis and management of patients with inherited primary arrhythmia syndromes. Heart Rhythm: the Official Journal of the Heart Rhythm Society, 10, 1932-1961. https://doi.org/10.1016/j. hrthm.2013.05.014

Qu, Z., Xie, Y., Garfinkel, A., \& Weiss, J. N. (2010). T-wave alternans and arrhythmogenesis in cardiac diseases. Frontiers in Physiology, 1, 154. https://doi.org/10.3389/fphys.2010.00154

Rashba, E. J., Cooklin, M., MacMurdy, K., Kavesh, N., Kirk, M., Sarang, S., ... Gold, M. R. (2002). Effects of selective autonomic blockade on T-wave alternans in humans. Circulation, 105, 837-842. https://doi. org/10.1161/hc0702.104127

Schomer, A. C., Nearing, B. D., Schachter, S. C., \& Verrier, R. L. (2014). Vagus nerve stimulation reduces cardiac electrical instability assessed by quantitative T-wave alternans analysis in patients with drug-resistant focal epilepsy. Epilepsia, 55, 1996-2002. https://doi. org/10.1111/epi.12855

Schwartz, P. J., Crotti, L., \& Insolia, R. (2012). Long-QT syndrome: From genetics to management. Circulation: Arrhythmia and Electrophysiology, 5, 868-877. https://doi.org/10.1161/CIRCEP.111.962019

Schwartz, P. J., \& Malliani, A. (1975). Electrical alternation of the T-wave: Clinical and experimental evidence of its relationship with the sympathetic nervous system and with the long Q-T syndrome. American Heart Journal, 89, 45-50. https://doi.org/10.1016/0002-8703(75)90008-3

Schwartz, P. J., Periti, M., \& Malliani, A. (1975). The long Q-T syndrome. American Heart Journal, 89, 378-390. https://doi. org/10.1016/0002-8703(75)90089-7

Shimizu, W., \& Antzelevitch, C. (1999). Cellular and ionic basis for T-wave alternans under long-QT conditions. Circulation, 99, 1499-1507. https://doi.org/10.1161/01.CIR.99.11.1499

Sugiyama, H., Tachibana, M., Morita, H., Nishii, N., Miyoshi, A., Sugiyama, H., ... Ito, H. (2017). Epicardially placed implantable cardioverter-defibrillator for a child with congenital long QT syndrome. Journal of Arrhythmia, 33, 237-239. https://doi.org/10.1016/j.joa.2016.10.561

Taggart, P., Sutton, P., Chalabi, Z., Boyett, M. R., Simon, R., Elliott, D., \& Gill, J. S. (2003). Effect of adrenergic stimulation on action potential duration restitution in humans. Circulation, 107, 285-289. https://doi. org/10.1161/01.CIR.0000044941.13346.74

Takasugi, N., Goto, H., Takasugi, M., Verrier, R. L., Kuwahara, T., Kubota, T., ... Minatoguchi, S. (2016). Prevalence of microvolt T-wave alternans in patients with Long QT Syndrome and its association with Torsade de Pointes. Circulation: Arrhythmia and Electrophysiology, 9, e003206. https://doi.org/10.1161/CIRCEP.115.003206

Takasugi, N., Takasugi, M., Goto, H., Kuwahara, T., Nakashima, T., Kubota, T., ... Verrier, R. L. (2018). Significance of T-wave inversion triggered by spontaneous atrial premature beats in patients with long QT 
syndrome. Heart Rhythm: the Official Journal of the Heart Rhythm Society, S1547-5271(18), 30098-30105. https://doi.org/10.1016/j. hrthm.2018.02.003

Verrier, R. L. (2018). The power of the patch: A smart way to track risk for torsades de pointes in congenital and drug-induced long QT syndromes? International Journal of Cardiology, 266, 145-146.

Verrier, R. L., \& Ikeda, T. (2013). Ambulatory ECG-based T-wave alternans monitoring for risk assessment and guiding medical therapy: Mechanisms and clinical applications. Progress in Cardiovascular Diseases, 56, 172-185. https://doi.org/10.1016/j.pcad.2013.07.002

Verrier, R. L., Klingenheben, T., Malik, M., El-Sherif, N., Exner, D. V., Hohnloser, S. H., ... Rosenbaum, D. S. (2011). Microvolt T-wave alternans: Physiologic basis, methods of measurement, and clinical utility. Consensus guideline by the International Society for Holter and Noninvasive Electrocardiology. Journal of the American College of Cardiology, 44, 1309-1324. https://doi.org/10.1016/j.jacc.2011.06.029

Vincent, G. M., Schwartz, P. J., Denjoy, I., Swan, H., Bithell, C., Spazzolini, C., ... Zhang, L. (2009). High efficacy of beta-blockers in long-QT syndrome type 1: Contribution of noncompliance and QT-prolonging drugs to the occurrence of beta-blocker treatment "failures.". Circulation, 119, 215-221.

Wilde, A. A., Moss, A. J., Kaufman, E. S., Shimizu, W., Peterson, D. R., Benhorin, J., ... Ackerman, M. J. (2016). Clinical Aspects of Type 3 LongQT Syndrome: An International Multicenter Study. Circulation, 134, 872-882. https://doi.org/10.1161/CIRCULATIONAHA.116.021823

How to cite this article: Takasugi N, Takasugi M, Goto $\mathrm{H}$, Kuwahara T, Kawasaki M, Verrier RL. Effect of beta-blockade on quantitative microvolt T-wave alternans in 24-hour continuous 12-lead ECG recordings in patients with long QT syndrome. Ann Noninvasive Electrocardiol. 2019;24:e12640. https://doi.org/10.1111/anec.12640 